\title{
Levantamento fitossociológico como ferramenta para a restauração florestal da Mata Atlântica, no Médio Paraíba do Sul
}

\author{
Phytosociological survey as a tool for the Atlantic Forest restoration in Médio \\ Paraíba do Sul
}

\author{
Cristiana do Couto Miranda ${ }^{\mathrm{I}}$, Alexandre De Donato ${ }^{\mathrm{II}}$, \\ Pablo Hugo Alves Figueiredo ${ }^{\mathrm{III}}$, Thiago Andrade Bernini ${ }^{\mathrm{IV}}$, Cristiane Roppa ${ }^{\mathrm{v}}$, \\ Isabela Bandeira Trece ${ }^{\mathrm{VI}}$, Letícia Oliveira Barros ${ }^{\mathrm{VII}}$
}

\begin{abstract}
Resumo
Levantamentos fitossociológicos constituem uma ferramenta de suporte à tomada de decisão em projetos de restauração florestal, contribuindo com a manutenção da biodiversidade vegetal e com o aumento da qualidade dos serviços prestados por esses ecossistemas. O presente estudo teve como objetivo realizar o levantamento fitossociológico das formações florestais presentes no Espaço Ecológico Educativo, Pinheiral-RJ e, com base nos resultados, discutir ações necessárias para restauração dos remanescentes. Foram instaladas parcelas retangulares de $20 \times 10 \mathrm{~m}$, distribuídas arbitrariamente nas florestas de encosta e aluvial, totalizando 0,3 ha de área amostral e cerca de $5,0 \%$ da área florestal. Todos os indivíduos com Diâmetro a Altura do Peito (DAP) $>5,0 \mathrm{~cm}$ foram registrados. A floresta aluvial apresentou 27 espécies e densidade de 1.600 ind./ha, enquanto na de encosta foram 37 espécies e densidade de 1.393 ind./ha. O índice de Shannon foi 2,98 e a equabilidade foi 0,56 para floresta de encosta e, respectivamente 2,59 e 0,48, para floresta aluvial. A composição florística variou significativamente entre as duas formações florestais analisadas, as quais apresentaram baixos valores de riqueza e diversidade de espécies, demonstrando a necessidade de enriquecimento com espécies florestais, na tentativa de restabelecer os valores de biodiversidade encontrados em ecossistemas florestais de referência.
\end{abstract}

Palavras-chave: Enriquecimento florestal; Floresta Aluvial; Floresta de Encosta; Médio Paraíba do Sul

\begin{abstract}
Phytosociological surveys may consist as decision-making supports for implementation of projects aimed at maintaining the biodiversity or increasing the quality of services provided by the ecosystems. The present study was an immediate making the phytosociological survey of forest formation present at 'Espaço Ecológico Educativo', Pinheiral - RJ state, and based on the results, discuss actions needed to restore the remaining. Rectangular plots of $20 \times 10 \mathrm{~m}$ were installed and distributed randomly in the slopes and alluvial forests, totaling 0.3 ha of the sampling area, about $5.0 \%$ of the forest. All the individuals with Diameter at Breast Height $(\mathrm{DBH})>5.0 \mathrm{~cm}$ were recorded. The alluvial forests showed 27 species and density of 1600 ind./ha while in the slope were 37 species and the density of 1393 ind./ha. The Shannon index was 2.98 and the evenness was 0.56 for the forest in the slope and for alluvial forest the diversity was 2.59 and the evenness was 0.48 . The floristic composition varied significantly between the forest types. The forest formations present in the area had low richness and diversity of species, demonstrating the need for enrichment in an attempt to restore the biodiversity values found in the reference forest ecosystems.
\end{abstract}

Keywords: Forest enrichment; Aluvial Forest; Slope Forest; Médio Paraíba do Sul

Bióloga, DSc., Professora da Pós-graduação em Desenvolvimento Regional e Sustentabilidade, Instituto Federal de Educação, Ciência e Tecnologia, Rua José Breves, 550, CEP 27197-000, Pinheiral (RJ), Brasil. cristiana.miranda@ifrj.edu.br (ORCID: 0000-0003-0725-0371)

II Engenheiro Agrônomo, MSc., Mestre em Fitossanidade e Biotecnologia Aplicada, Instituto Federal de Educação, Ciência e Tecnologia, Rua José Breves, 550, CEP 27197-000, Pinheiral (RJ), Brasil. alexandre.donato@ifrj.edu.br (ORCID: 0000-0002-6514-2654)

III Engenheiro Florestal, MSc, Doutorando em Botânica, Escola Nacional de Botânica Tropical, Rua Pacheco Leão 2040, CEP 22460-030, Rio de Janeiro (RJ), Brasil. pablo_figueiredo@yahoo.com.br (ORCID: 0000-0003-4133-7315)

IV Engenheiro Agrônomo, DSc. Doutor em Recursos Naturais e Proteção Ambiental, Instituto Federal de Educação, Ciência e Tecnologia, Rua José Breves, 550, CEP 27197-000, Pinheiral (RJ), Brasil. thiago.bernini@ifrj.edu.br (ORCID: 0000-0001-6476-5000)

Engenheira Florestal, DSc., Doutora em Ciências Ambientais e Florestais, Pesquisadora Autônoma, Rua professora Ana Ferreira de Oliveira, 47, CEP 27410-270, Quatis (RJ), Brasil. cristianeroppa@gmail.com (ORCID: 0000-0002-3902-4662)

VI Técnica em Meio Ambiente, Graduanda em Engenharia Florestal, Universidade Federal Rural do Rio de Janeiro, Rodovia BR 465 km 07 s/n, CEP 23890-000, Seropédica (RJ), Brasil. isabelabtrece@gmail.com (ORCID: 0000-0002-2873-6963)

viI Técnica em Meio Ambiente, Graduanda em Ciências Biológicas, Universidade Federal do Rio de Janeiro, Av. Pedro Calmon, 550, CEP 21941-901, Rio de Janeiro (RJ), Brasil. leticiabarrosifrj@gmail.com (ORCID: 0000-0002-6657-9751) 


\section{Introdução}

A descrição de comunidades vegetais apresenta propósitos comparativos, com vistas a revelar padrões de variação da vegetação no espaço e no tempo, consistindo em uma importante ferramenta para o reconhecimento da estrutura das comunidades vegetais e a relação com o ambiente no qual estão inseridas (DURINGA et al., 2016; EISENLOHR et al., 2011). Por este motivo, a fitossociologia vem sendo um dos ramos da ecologia vegetal mais utilizados para o reconhecimento das relações quantitativas entre as espécies, principalmente de comunidades arbóreas (EISENLOHR et al., 2011).

A partir das informações geradas por esses estudos, é possível desenvolver ações mais eficientes no sentido de atenuar as consequências da perda de biodiversidade (TARGA et al., 2017), além de subsidiar planos para recuperação dos ecossistemas florestais (ROSA et al., 2008). Portanto, é essencial que sejam realizados levantamentos florísticos na própria área de trabalho, com a finalidade de caracterizar os fragmentos remanescentes, no que se refere ao tipo vegetacional, às espécies ocorrentes, e à classificação quanto ao estado atual de conservação. Essa caracterização deve ser realizada em cada um dos fragmentos florestais da região abrangida, visando à maior adequação de metodologias e ações de restauração, tanto do próprio fragmento, como das áreas do entorno imediato (SILVA et al., 2017).

Em regiões cujos remanescentes florestais se encontram altamente fragmentados, levantamentos fitossociológicos podem dar suporte à tomada de decisão pelos administradores públicos e/ou privados, para realização de projetos que visem à manutenção da biodiversidade ou ao aumento da qualidade dos serviços prestados por esses ecossistemas (SILVA et al., 2017). Exclusivamente no território fluminense, onde foram registrados 30,6\% de remanescentes florestais, compreendendo $22 \%$ de Floresta Ombrófila Densa e 5\% de Floresta Estacional Semidecidual (BRASIL, 2014), municípios como Pinheiral, situado na Região do Médio Paraíba do Sul, que conta com menos de 1.000 ha de florestas (RIO DE JANEIRO, 2014), necessitam urgentemente dessas ações.

O município de Pinheiral sintetiza a história de ocupação da bacia hidrográfica do rio Paraíba do Sul, em especial do Médio Vale do rio Paraíba. Os graves problemas oriundos desse histórico de ocupação e uso do solo repercutiram na intensa fragmentação florestal da região (HONJI et al.,2017). De acordo com o Tribunal de Contas do Estado do Rio de Janeiro (TCE-RJ, 2004), $18 \%$ da área do Município de Pinheiral correspondem à vegetação secundária e $76 \%$ é ocupada por pastagens degradadas, nativas e plantadas. Grande parte dessas áreas se encontra com tendência de degradação, ou seja, empobrecimento paulatino do solo, mesmo com mínimas taxas de uso (MIRANDA, 2011).

A área de Pinheiral apresenta graves problemas de erosão, o que traz prejuízos econômicos e socioambientais, com ênfase no que tange à produção de sedimentos e sua transferência para o rio Paraíba do Sul, principal transposição para formação do Rio Guandu (fonte de abastecimento para a região metropolitana do Rio de Janeiro). (GAIA-GOMES et al., 2017) Segundo o Comitê para Integração da Bacia Hidrográfica do Rio Paraíba do Sul (CEIVAP, 2002), 88 \% da área do município está na classe de alta ou muito alta vulnerabilidade à erosão. As queimadas, que têm ocorrido em diversas áreas do município, agravam ainda mais esta vulnerabilidade.

Apesar desse contexto, o que se observa é uma relativa inércia da sociedade frente às questões de degradação ambiental e perda da biodiversidade. Para reverter esse quadro atual, é necessário um esforço conjunto da sociedade, sendo que a ciência e a educação ambiental têm papéis especiais. Pautado nesta necessidade, foi criado o Espaço Ecológico Educativo (EEcoE), no Instituto Federal do Rio de Janeiro (IFRJ), Campus Pinheiral, com objetivo de realizar pesquisas que visem à conservação e à restauração de florestas, priorizando fragmentos florestais existentes, e promover ações de educação ambiental para a comunidade do município de Pinheiral e da região.

No intuito de subsidiar ações de restauração florestal que possam servir de modelos 
para as demais áreas da Região do Médio Paraíba do Sul, o presente estudo teve como objetivo realizar o levantamento fitossociológico das formações florestais contidas no EEcoE e, com base nos resultados, discutir ações necessárias para a restauração dos remanescentes.

\section{Material e método}

\section{Área de estudo}

A área de estudo, denominada EEcoE, é um laboratório ao ar livre do IFRJ - Pinheiral (município de Pinheiral, Rio de Janeiro), representativo das condições ambientais, históricas e geomorfológicas da região, que pode ser usado como uma área demonstrativa permanente, contribuindo na propagação do conhecimento formal e não formal da conservação e restauração florestal. Essa área é delimitada pelas coordenadas UTM Zona 235, $7508700 \mathrm{~N}$ e $603400 \mathrm{~L}$, compreendendo uma área de 37 ha.

O mapeamento do uso do solo demonstrou que o EEcoE é composto por $41,4 \%$ de pasto sujo, $37,8 \%$ de capoeira, $20,8 \%$ de floresta e $7,0 \%$ de solo exposto (Figura 1). A classe de floresta está dividida em duas formações florestais: as situadas nas encostas - Floresta Estacional Semidecidual Submontana (IBGE, 2012) - e outra nas áreas de planície sobre influência do lençol freático, sujeita a alagamentos temporários - Floresta Estacional Semidecidual Aluvial (IBGE, 2012).

Figura 1 - Mapa de uso do solo do Espaço ecológico Educativo (EEcoE), em Pinheiral-RJ.

Figure 1 - Land-use map of Educational Ecological Space, in Pinheiral-RJ state.

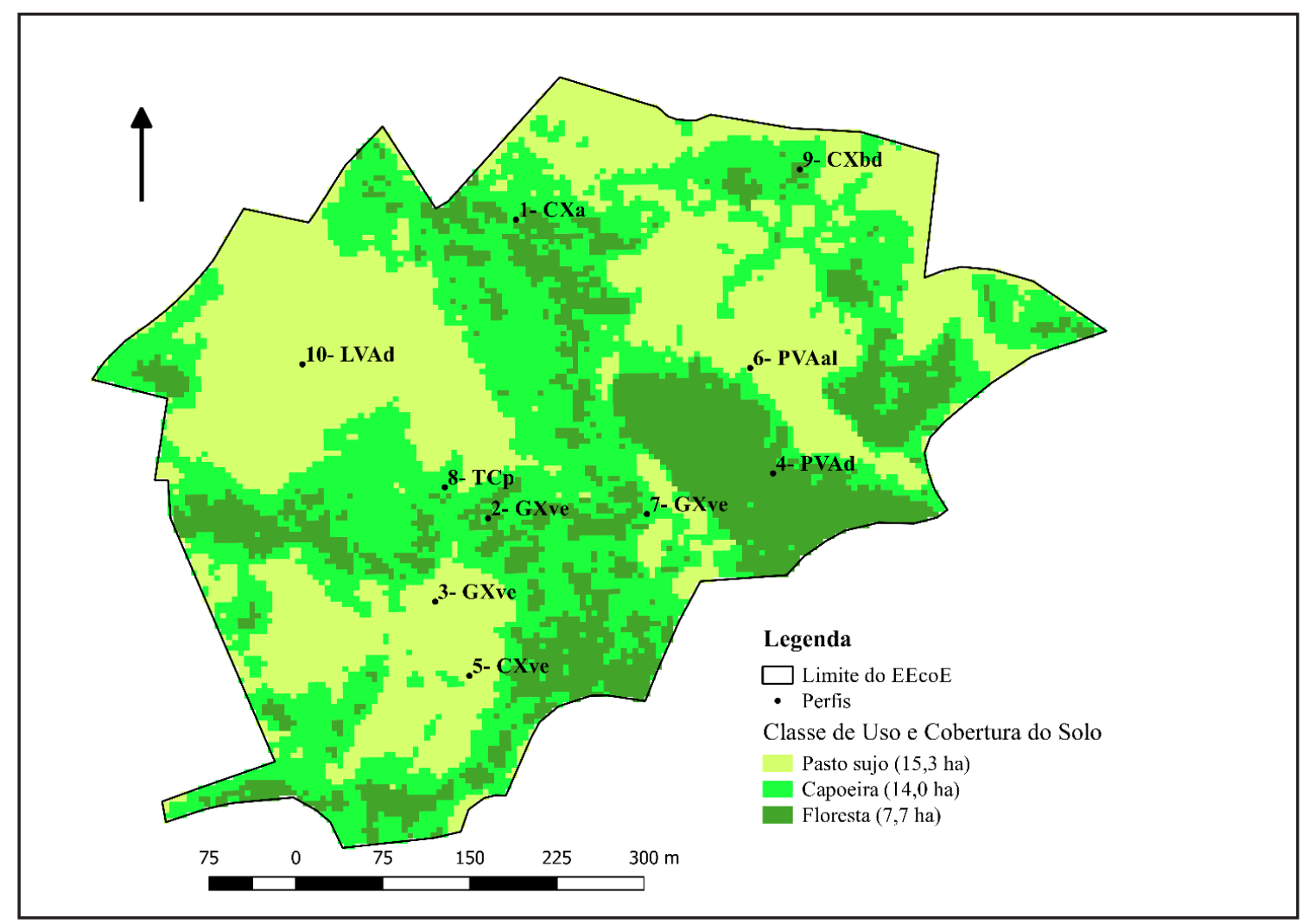

Fonte: Autores (2019) 
O clima da região, de acordo com a classificação de Köppen, foi identificado de duas formas distintas, em Cwa - clima temperado de inverno seco e verão chuvoso, e Am - clima tropical chuvoso com inverno seco (OLIVEIRA, 1998). Dados da antiga estação meteorológica de Piraí, apresentados por Oliveira (1998), indicam que a região apresenta precipitação anual em torno de $1308 \mathrm{~mm}$ e temperatura média anual de $20,9^{\circ} \mathrm{C}$.

\section{Coleta e análise de dados}

Para amostragem da área, foram instaladas 15 parcelas retangulares de $20 \times 10 \mathrm{~m}\left(200 \mathrm{~m}^{2}\right)$, orientadas no sentido norte, distribuídas aleatoriamente nas duas formações florestais. Ao todo foram 8 parcelas na floresta de encosta e 7 na aluvial, totalizando 0,3 ha de área amostral, cerca de 5,0 \% da área florestal do EEcoE. Todos os indivíduos com DAP $>5,0 \mathrm{~cm}$ dentro da parcela tiveram sua circunferência registradas e alturas estimadas.

A grafia dos nomes científicos e a checagem de sinônimos foram realizadas com base na lista de espécies da Flora do Brasil 2020 em construção (2016). A classificação das famílias foi baseada no sistema APGIV (Angiosperm Phylogeny Group) (CHASE et al., 2016).

Todos os indivíduos amostrados e identificados em nível de espécie foram classificados em grupos baseado nos dados da biologia e autoecologia disponíveis na literatura, conforme os seguintes grupos ecológicos: pioneiras (PI), secundárias iniciais (SI), secundárias tardias (ST) e não classificadas (NC) (GANDOLFI et al., 1995); síndrome de dispersão: anemocóricas, zoocóricas, autocóricas e não classificadas (VAN DER PIJL, 1982).

Para cada espécie, foram calculados os parâmetros fitossociológicos: densidade, dominância (absoluta e relativa) e frequência (absoluta e relativa). A partir desses, foram calculados os índices de valor de cobertura e de valor de importância (MUELLER-DOMBOIS; ELLENBERG, 1974).

A diversidade de espécies, para cada formação, foi estimada através do índice de Shannon (H’) (MAGURRAN, 1988). Na comparação da similaridade florística, foi utilizada a análise de agrupamento pelo método completo, que considera a distância entre os vizinhos mais distantes como a distância entre agrupamentos, a partir de uma matriz de abundância das espécies, gerando uma matriz de coeficientes de distâncias euclidiana (GOTELLI; ELLISON, 2011). Para testar a significância da diferença de composição florística entre as formações florestais, foi utilizada a Análise de Similaridade (ANOSIM), através do pacote estatístico Vegan, do programa $\mathrm{R}$ (OKSANEN et al., 2017). Esta análise é considerada análoga a ANOVA, porém, utiliza permutação das amostras e compara a similaridade entre matrizes de distância (OKSANEN et al.,2017).

\section{Resultados e discussão}

Foram registradas 57 espécies distribuídas em 50 gêneros e 27 famílias botânicas. (Tab. 1). As famílias mais ricas situadas na floresta aluvial foram: Fabaceae (7 espécies), Myrtaceae (4) e Sapindaceae (2), enquanto na floresta de encosta, a família Fabaceae (12 espécies) foi predominante e as demais com duas espécies cada. A densidade média total foi equivalente a 1.140 ind./ha, sendo que, desta estimativa total, 80 ind/ha estavam mortos. Levando em consideração os dois ambientes separados, a floresta aluvial apresentou 27 espécies e densidade equivalente a 1.600 ind./ha, enquanto a de encosta 37 espécies e cerca de 1.393 ind./ha, conforme a Tabela 1 que segue abaixo:

As espécies nativas com maior valor de importância na floresta de encosta foram Piptadenia gonoacantha (46,7), Sparattosperma leucanthum (26,6), Pseudopiptadenia contorta (25,3), Apuleia leiocarpa $(24,8)$ e Cupania oblongifolia $(20,5)$. Os indivíduos mortos apresentaram alto valor de importância $(30,6)$, proporcionado pelos elevados valores de densidade $(10 \%)$ e dominância relativas (12\%). Na floresta aluvial, as espécies com maiores valores de importância foram Clitoria fairchildiana $(49,3)$, Guarea guidonia $(47,3)$, Inga striata $(27,5)$, Casearia sylvestris $(19,7)$ e Citharexylum myrianthum $(18,4)$. 
Tabela 1 - Lista florística e número de indivíduos registrados nas formações florestais situadas na planície e encosta do Espaço Ecológico Educativo, em Pinheiral-RJ.

Table 1 - Floristic list and number of individuals recorded in the forest formation located in the plains and hills of 'Espaço Ecológico Educativo', in Pinheiral-RJ.

\begin{tabular}{|c|c|c|c|}
\hline Família/Espécie & Planície & Encosta & Total \\
\hline Anacardiaceae & 16 & 3 & 19 \\
\hline Astronium fraxinifolium Schott & & 3 & 3 \\
\hline Schinus terebinthifolius Raddi & 16 & & 16 \\
\hline Annonaceae & & 3 & 3 \\
\hline Guatteria candolleana Schltdl. & & 3 & 3 \\
\hline Apocynaceae & 2 & & 2 \\
\hline Tabernaemontana laeta Mart. & 2 & & 2 \\
\hline Arecaceae & & 7 & 7 \\
\hline Astrocaryum aculeatissimum (Schott) Burret & & 6 & 6 \\
\hline Syagrus romanzoffiana (Cham.) Glassman & & 1 & 1 \\
\hline Bignoniaceae & & 13 & 13 \\
\hline Cybistax antisyphilitica (Mart.) Mart. & & 1 & 1 \\
\hline Sparattosperma leucanthum (Vell.) K.Schum. & & 12 & 12 \\
\hline Boraginaceae & & 1 & 1 \\
\hline Cordia sp. & & 1 & 1 \\
\hline Cannabaceae & 1 & & 1 \\
\hline Trema micrantha $($ L.) Blume & 1 & & 1 \\
\hline Erythroxylaceae & & 2 & 2 \\
\hline Erythroxylum pulchrum A.St.-Hil. & & 2 & 2 \\
\hline Euphorbiaceae & 2 & & 2 \\
\hline Croton urucurana Baill. & 2 & & 2 \\
\hline Fabaceae & 46 & 76 & 122 \\
\hline Albizia polycephala (Benth.) Killip ex Record & & 7 & 7 \\
\hline Apuleia leiocarpa (Vogel) J.F.Macbr. & & 23 & 23 \\
\hline Centrolobium sp. & & 1 & 1 \\
\hline Clitoria fairchildiana R.A.Howard & 14 & & 14 \\
\hline Dalbergia frutescens (Vell.) Britton & & 1 & 1 \\
\hline Erythrina speciosa Andrews & 10 & & 10 \\
\hline Inga striata Benth. & 9 & 3 & 12 \\
\hline Libidibia ferrea (Mart. Ex Tul.) L.P.Queiroz & & 1 & 1 \\
\hline Machaerium hirtum (Vell.) Stellfeld & 3 & & 3 \\
\hline Machaerium nyctitans (Vell.) Benth. & & 1 & 1 \\
\hline
\end{tabular}


Tabela 1 - Continuação ...

Table 1 - Continuation ...

\begin{tabular}{|c|c|c|c|}
\hline Família/Espécie & Planície & Encosta & Total \\
\hline Machaerium sp.3 & & 1 & 1 \\
\hline Mimosa artemisiana Heringer \& Paula & 4 & 1 & 5 \\
\hline Mimosa bimucronata (DC.) Kuntze & 4 & & 4 \\
\hline Piptadenia gonoacantha (Mart.) J.F.Macbr. & 2 & 18 & 20 \\
\hline Piptadenia paniculata Benth. & & 3 & 3 \\
\hline $\begin{array}{l}\text { Pseudopiptadenia contorta (DC.) } \\
\text { G.P.Lewis\&M.P.Lima }\end{array}$ & & 16 & 16 \\
\hline Lauraceae & 1 & & 1 \\
\hline Nectandra membranacea (Sw.) Griseb. & 1 & & 1 \\
\hline Lecythidaceae & & 1 & 1 \\
\hline Lecythis sp. & & 1 & 1 \\
\hline Malvaceae & & 4 & 4 \\
\hline Eriotheca sp. & & 1 & 1 \\
\hline Luehea grandiflora Mart. \&Zucc. & & 3 & 3 \\
\hline Melastomataceae & 1 & & 1 \\
\hline Miconia prasina $($ Sw. $)$ DC. & 1 & & 1 \\
\hline Meliaceae & 43 & & 43 \\
\hline Guarea guidonia (L.) Sleumer & 43 & & 43 \\
\hline Moraceae & 1 & 3 & 4 \\
\hline Brosimum guianense (Aubl.) Huber & & 3 & 3 \\
\hline Maclura tinctoria (L.) D. Don ex Steud. & 1 & & 1 \\
\hline Myrtaceae & 9 & 3 & 12 \\
\hline Campomanesia sp. & & 2 & 2 \\
\hline Eugenia florida DC. & 4 & & 4 \\
\hline Myrcia splendens (Sw.) DC. & 1 & 1 & 2 \\
\hline Psidium guajava $\mathrm{L}$. & 2 & & 2 \\
\hline Syzygium cumini (L.) Skeels & 2 & & 2 \\
\hline Nyctaginaceae & 1 & 4 & 5 \\
\hline Guapira opposita (Vell.) Reitz & 1 & 4 & 5 \\
\hline Phyllantaceae & & 1 & 1 \\
\hline Margarita rianobilis L.f. & & 1 & 1 \\
\hline Rutaceae & & 1 & 1 \\
\hline Zanthoxylum rhoifolium Lam. & & 1 & 1 \\
\hline Salicaceae & 20 & 13 & 33 \\
\hline Casearia sylvestris Sw. & 20 & 13 & 33 \\
\hline
\end{tabular}


Tabela 1 - Conclusão ...

Table 1 - Conclusion ...

\begin{tabular}{|c|c|c|c|}
\hline Família/Espécie & Planície & Encosta & Total \\
\hline Sapindaceae & 3 & 17 & 20 \\
\hline Cupania oblongifolia Mart. & 2 & 16 & 18 \\
\hline Matayba juglandifolia Radlk. & & 1 & 1 \\
\hline Sapindus saponaria $\mathbf{L}$. & 1 & & 1 \\
\hline Siparunaceae & 1 & & 1 \\
\hline Siparuna guianensis Aubl. & 1 & & 1 \\
\hline Solanaceae & 5 & & 5 \\
\hline Aureliana sp. & 4 & & 4 \\
\hline Solanum sp. & 1 & & 1 \\
\hline Urticaceae & & 4 & 4 \\
\hline Cecropia hololeuca Miq. & & 2 & 2 \\
\hline Cecropia pachystachya Trécul & & 2 & 2 \\
\hline Verbenaceae & 5 & & 5 \\
\hline Citharexylum myrianthum Cham. & 5 & & 5 \\
\hline
\end{tabular}

Fonte: Autores (2019)

Em relação ao grupo ecológico, na floresta de encosta a maior proporção corresponde às secundárias iniciais (76\%), seguida das pioneiras (17\%) e secundárias tardias (7\%). Na floresta aluvial, 14 espécies foram classificadas como pioneiras e 13 como secundárias iniciais. Em relação à densidade, os dois grupos apresentaram proporções muito similares, com $50 \%$ para as secundárias iniciais e $45 \%$ para as pioneiras. No entanto, a dominância das pioneiras foi o dobro das secundárias iniciais, equivalente a $9,23 \mathrm{~m}^{2} /$ ha. Este acontecimento se deve ao fato de cerca de $70 \%$ das secundárias iniciais apresentarem diâmetro médio de $7,5 \mathrm{~cm}$, demonstrando que a maior parte dessas se encontra no estrato regenerativo.

$\mathrm{Na}$ floresta de encosta, 9 espécies são anemocóricas, 7 autocóricas e 17 zoocóricas. Os indivíduos zoocóricos e autocóricos apresentaram a mesma proporção, $42,5 \%$. No entanto, a dominância das espécies autocóricas foi muito maior que das zoocóricas, $15,1 \mathrm{~m}^{2} / \mathrm{ha} \mathrm{e} 5,0 \mathrm{~m}^{2} /$ ha, respectivamente. Na floresta aluvial, 20 espécies são zoocóricas, 9 autocóricas e apenas 1 é anemocórica. Os indivíduos zoocóricos representaram 70,5\%, porém, sua dominância foi equivalente à dos indivíduos autocóricos, ambos com aproximadamente $6,5 \mathrm{~m}^{2} / \mathrm{ha}$.

O índice de Shannon foi 2,98 e a equabilidade 0,56, para floresta de encosta e 2,59 e 0,48, respectivamente para floresta aluvial. A composição florística variou significativamente entre os dois ambientes de floresta $(R=0,962 ; \mathrm{p}=0,001)$.

$\mathrm{Na}$ floresta de encosta, a dominância das espécies autocóricas ocorreu porque a maior parte delas é representada por espécies da família Fabaceae, entre elas, Piptadenia paniculata, Apuleia leiocarpa e Piptadenia gonoacantha, que são geralmente emergentes (ROTH, 1987; YAMAMOTO et al., 2007; LOPES et al., 2014), e que, por isso, atingiram grandes dimensões diamétricas, com cerca de $30 \%$ dos indivíduos apresentando diâmetro médio maior que 25 cm. Já as zoocóricas encontradas, são geralmente espécies de sub-bosque, ou no máximo de dossel (ROTH, 1987; YAMAMOTO et al., 2007; LOPES et al., 2014), que, quando comparadas às emergentes, apresentaram diâmetro muito inferior, com cerca de $65 \%$ dos indivíduos com diâmetro médio de $7,5 \mathrm{~cm}$, das quais as mais representativas foram Cupania oblongifolia e Casearia 
sylvestris.

As duas formações apresentaram elevado valor de espécies exclusivas, sendo o maior valor para a floresta de encosta, $81 \%$ das espécies, destacando-se espécies como Pseudopiptadenia contorta, Apuleia leiocarpa, Sparattosperma leucanthum e Albizia polycephala. Na formação aluvial, $74 \%$ espécies foram exclusivas, das quais as mais abundantes foram Guarea guidonia, Schinus terebinthifolius, Citharexylum myrianthum e Croton urucurana. As sete espécies generalistas foram Cupania oblongifolia, Inga striata, Guapira opposita, Piptadenia gonoacantha, Casearia sylvestris, Myrcia artemisiana e Myrcia splendens. Os resultados da ocorrência de espécies exclusivas de cada formação, indicam que os dois ambientes apresentam particularidades florísticas definidas pelas características locais.

Através da análise fitossociológica, ficou evidente que as duas formações florestais apresentam elevado grau de perturbação, elucidado pelo baixo número de espécies e pela diversidade florística. No entanto, a alta densidade de secundárias iniciais, com DAP médio de $7,5 \mathrm{~cm}$, sugere que estas estão sendo recrutadas. Portanto, é possível que esteja ocorrendo o processo de sucessão natural, pois há indícios de que espécies mais exigentes quanto às condições ambientais estejam aos poucos substituindo as pioneiras (BUDOWISK, 1965; GANDOLFI et al.,1995).

Apesar desta tendência, tanto a floresta de encosta quanto a aluvial precisam ser enriquecidas, ou seja, devem ser introduzidas espécies dos estágios finais de sucessão, especialmente aquelas de maior interação com a fauna, e/ou das diversas formas vegetais originais de cada formação florestal, com objetivo de restabelecer seus processos naturais e funcionalidade através do aumento da diversidade florística (ROZZA et al., 2006). Ao comparar os valores obtidos nestas áreas com ecossistemas referenciais (florestas em bom estado de conservação), esta afirmação fica evidente.

Em trechos de Florestas Semideciduais preservadas, na Estação Ecológica de Caetetus, a densidade foi equivalente a $1.080 \mathrm{ind} / \mathrm{ha}$ e a riqueza de 62 espécies (DURIGAN et al., 2016). Enquanto de acordo com os resultados apresentados por Leite e Rodrigues (2008) no Parque Municipal de São Roque, a densidade foi de 1.495,24 ind./ha, no qual foram encontradas 117 espécies e 47 famílias. Em estudo efetuado em dez fragmentos de florestas semideciduais no extremo oeste de Minas Gerais, Lopes et al. (2012) observaram que a diversidade vegetal variou de 2,92 a 3,97. Já nos fragmentos estudados por Abreu et al. (2014), na região norte do Rio de Janeiro, o índice chegou a 3,8 .

A superior densidade observada na floresta aluvial, em comparação com a floresta de encosta, não é suficiente para afirmar que tal parâmetro se encontra dentro da normalidade esperada para essa formação florestal, visto que, Ivanauskas et al. (1997) e Sztutman e Rodrigues (2002), ao analisarem um ecossistema semelhante, observaram uma densidade variando dentro da amplitude de $1.310 \mathrm{ind} / \mathrm{ha}$ e $2730 \mathrm{ind} / \mathrm{ha}$, em função de variáveis como profundidade do solo e tempo de inundação. Outro parâmetro que pode ser considerado abaixo do padrão ideal para a floresta aluvial é a diversidade, considerando que Carvalho et al., (2006) encontraram 4,1 para uma floresta de baixada aluvial, na região norte do Rio de Janeiro, enquanto no estudo de Ivanauskas et al. (1997), citado anteriormente, foi encontrado o valor de 3,0.

Um outro parâmetro a ser considerado é a riqueza de espécies, que de acordo com alguns estudos, mesmo em florestas bem conservadas, é inferior nas florestas de baixada em comparação às florestas com baixa influência do lençol freático, sendo reportadas 44 espécies em estudo de Teixeira e Assis (2005), em Rio Claro-SP e 40 espécies em levantamento feito por Marques et al. (2003), na cidade de Brotas-SP.

Pode-se afirmar que este padrão era esperado, já que relativamente poucas espécies arbóreas tropicais desenvolveram mecanismos adaptativos que permitam a sua sobrevivência em condições anaeróbicas (JOLY, 1991). Durante o processo evolutivo, o estresse causado pela saturação hídrica do solo, deve ser o principal fator atuante na seleção de espécies nas áreas inundáveis (LOBO; JOLY, 2000; IVANAUSKAS et al., 1997). Portanto, a combinação entre o grau 
de alagamento e a tolerância das espécies ali presentes condicionará a composição e estrutura da vegetação em um determinado local (SILVA et al.,2012).

Neste sentido, devem-se implantar espécies diferentes para as duas formações, a fim de enriquecê-las e promover a restauração florestal. A família Fabaceae é característica de florestas Semideciduais (RIZZINI, 1997), contudo, como a mesma apresentou o maior valor de importância na área $(124,2)$, é essencial que seja feito um enriquecimento com espécies de outras famílias, como as Cedrela spp. (Meliaceae), Cariniana spp. (Lecytidaceae), Aspidosperma spp. (Apocynaceae), Handroanthus spp. (Bignoniaceae), Balfourodendron sp. (Rutaceae) e muitas outras, características e predominantes nestas vegetações (RIZZINI, 1997; IBGE 2012).

Já para a floresta aluvial, podem ser priorizadas espécies dos gêneros Calophyllum sp., Symphonia sp. (ambas Clusiaceae), Sebastiania spp. (Euphorbiaceae), Hedyosmum spp. (Chloranthaceae) e Tapirira spp. (Anacardiaceae), características de áreas com solo sobre forte influência do lençol freático (IVANAUSKAS et al., 1997; RIZZINI, 1997; SILVA et al., 2012). Vale ressaltar que muitos dos gêneros supracitados apresentam espécies ameaçadas, por serem amplamente exploradas economicamente (MARTINELLI et al., 2013), e que, por isso, devem ser priorizadas para o enriquecimento (ROZZA et al., 2006).

Outro aspecto importante que pode ser levado em consideração para o enriquecimento, é que a maior parte das zoocóricas da área em estudo apresentam frutos muito pequenos, sendo que, de acordo com Muscarella e Fleming (2007), esse tipo de fruto tem como agentes dispersores pássaros e morcegos. Neste sentido, é interessante que sejam priorizadas espécies de frutos grandes, já que estas apresentam relações com animais de grande porte, importantes para a manutenção do ecossistema florestal (VIDAL et al., 2013).

Além disso, o uso de poleiros artificiais como técnica para indução da regeneração nas áreas sem cobertura florestal no EEcoE poderia ser viável, visto que a elevada proporção de espécies $(40 \%)$ e indivíduos (35\%) zoocóricos pioneiros, poderia desencadear o processo de colonização em novas áreas.

Poleiros artificiais podem ser construídos com varas de bambus, postes de eucaliptos, caules de árvores mortas e outros materiais encontrados no próprio ambiente. Tal construção ecologicamente correta pode fortalecer atividades educativas voltadas à conscientização quanto à importância da reciclagem e reutilização de materiais (FONSECA, 2014). As construções presentes ao longo das trilhas ecológicas no interior do EEcoE estão em consonância com estes princípios.

Maciel et al. (2017) destacam a importância das trilhas ecológicas como uma poderosa ferramenta para educação ambiental, em virtude destas permitirem a exploração de diversas áreas do conhecimento, tais como: conservação, fauna, flora, geografia, geologia, ecologia, urbanismo, história, além de outras, pois além de observar os diversos fenômenos que ocorrem naquele meio, é possível trabalhar abstrações acerca dos impactos sofridos por determinadas áreas (resgate histórico ambiental), e como estes se relacionam com sua perspectiva de recuperação. Desta forma, se constituem em uma ferramenta de ensino experiencial altamente interdisciplinar que transcende a transmissão de conhecimento, proporcionando a sensibilização e o efetivo envolvimento do visitante.

O desenvolvimento de ações de educação ambiental em espaços não formais, como o EEcoE, é um importante aliado no processo de conservação dos ecossistemas naturais, assim como no resgate dos seus serviços. Chaves (2017) analisando o desenvolvimento das ações de educação ambiental pela escola Cabula I e buscando encontrar soluções para a degradação ambiental do horto florestal do Cabula, observou que é preciso uma interação participativa da comunidade, de forma a discutir os problemas e soluções conjuntamente. Magalhães et al. (2016) destacaram, em seu trabalho no horto florestal do Colégio Azevedo Amaral no Rio de Janeiro, a importância do envolvimento da comunidade do entorno no processo de conservação desse espaço. Isso pode ser estimulado, buscando alinhar pressupostos de educação ambiental crítica emancipatória, da educação popular e da pedagogia crítica. 


\section{Conclusão}

O levantamento fitossociológico indicou que os remanescentes florestais (floresta de encosta e aluvial) do EEcoE possuem baixos valores de riqueza e diversidade de espécies, necessitando de intervenções para acelerar a restauração florestal. Os dois ambientes apresentam particularidades florísticas, e para que tenham restabelecidos seus valores de biodiversidade, favorecendo a restauração florestal, recomenda-se o enriquecimento com espécies florestais, obedecendo ao tipo de ambiente de ocorrência de cada espécie, de maneira que as intervenções acarretem o menor número de perdas possível, e que as formações florestais passem a apresentar riqueza e composição florística semelhante às áreas naturais mais preservadas.

Devido à representatividade ambiental do EEcoE, as ações indicadas nesse estudo têm funções importantes em nível local e regional, pois contribuirão para a restauração do ecossistema florestal do EEcoE e poderão configurar-se em um modelo para outras ações de restauração florestal de áreas perturbadas semelhantes, as quais são numerosas na Região do Médio Paraíba do Sul, ressaltando a importância dessa área de pesquisa para a região. Dessa forma, esse espaço poderá contribuir na propagação do conhecimento formal e não formal da conservação e restauração florestal. Esse processo é potencializado, considerando que o EEcoE possui trilhas interpretativas, nas quais diferentes grupos escolares e sociais da região fazem visitas constantes. Essas visitas possuem o objetivo de estímulo à conscientização ambiental, ao ensino interdisciplinar e inclusão.

\section{Agradecimento}

Agradecemos ao técnico em Meio ambiente e Agropecuária, Almir Ferreira, que contribuiu para a realização dos trabalhos de campo.

\section{Referências}

ABREU, K.M.P. et al. Tree species diversity of coast allow land semi deciduous forest fragments in northern Rio de Janeiro State. Bioscience Journal, Uberlândia, v. 30, p. 1529-1550, set. 2014.

BUDOWISK, G. Distribution of tropical American rain Forest species in the light of successional processes. Turrialba, Costa Rica, v. 15, p. 40-42. 1965.

CARVALHO, F.A. et al. Comunidade arbórea de uma floresta de baixada aluvial no município de Campos dos Goytacazes, RJ. Cerne, Lavras, v. 12, p. 157-166. 2006.

CHASE, M. W. et al. An update of the Angiosperm Phylogeny Group classification for the orders and families of flowering plants: APG IV. Botanical Journal of the Linnean Society, [s.1], v. 181, n. 1, p. 1-20, 2016.

CHAVES, D.R. Educação Ambiental na Escola Municipal Cabula I: Processos de Valorização, Mobilização e Articulação entre Escola, Comunidade e Instituições Públicas em Prol do Horto Florestal do Cabula. In: 38 ${ }^{\mathbf{a}}$ Reunião Nacional do ANPEd. "Democracia em risco: a pesquisa e a pós-graduação em contexto de resistência”, 2017, São Luís, Anais eletrônicos, São Luís. Disponível em: http://38reuniao.anped.org.br/sites/default/files/resources/programacao/ trabalho_38anped_2017_GT22_1060.pdf. Acesso em: 20 jun. 2018.

CEIVAP - COMITÊ PARA INTEGRAÇÃO DA BACIA HIDROGRÁFICA DO RIO PARAÍBA DO SUL. Diagnóstico da situação atual dos recursos hídricos. In: Plano da bacia do Rio Paraíba do Sul. Vol. 1-8. Fundação COPPETEC, ANA, Rio de Janeiro. 1200p. 2002. Disponível em <http:// www.ceivap.org.br/planobacia.htm>. Acesso em 10 junho 2008.

DURIGAN, G. et al. Valores esperados para atributos de florestas ripárias em restauração em 
diferentes idades Expected values for atributes of riparian forests under going restoration at different ages. ScientiaForestalis, [s.l], v. 44, n. 110 p. 463-474, jun. 2016.

EISENLOHR, P.V. et al. Floresta Ombrófila Densa Atlântica: bases conceituais e estudo de caso no Parque Estadual Carlos Botelho, SP, Brasil. In: Felfili, J.M.; Eisenlohr, P.V.; Melo, M.M.R.F.; Andrade, L.A. \& Meira-Neto, J.A.A. (eds.). Fitossociologia no Brasil: métodos e estudos de casos. Editora UFV, Viçosa, p. 372-387. 2011.

FLORA DO BRASIL 2020 EM CONSTRUÇÃO. Lista de Espécies da Flora do Brasil. Jardim Botânico do Rio de Janeiro. Disponível em http://floradobrasil.jbrj.gov.br/. Acesso em 28 janeiro 2015.

FONSECA, G. M. R. Viveiro de mudas nativas: Um negócio Sustentável. 2014. 42f. Dissertação (Mestrado) - Pós-Graduação em Economia e Meio Ambiente, Universidade Federal do Paraná, Curitiba, 2014.

GANDOLFI, S.; LEITÃO-FILHO, H.F.; BEZERA, C.L.F. Levantamento florístico e caráter sucessional das espécies arbustivo-arbóreas de uma floresta mesófila semidecídua no município de Guarulhos, SP. Revista Brasileira de Biologia, [s.l], v. 55, p. 753-767. 1995.

GAIA-GOMES, J. H. et al. Physical parameters of the cachimbal river sub-basin associated to land scape change in Pinheiral - RJ, Brazil. BioscienceJournal, [s.l], v. 34, n. 2, 2018.

GOTELli, N. J.; ELlison, A. M. Princípios de estatística em ecologia. Porto Alegre, Artmed, 2011. $528 \mathrm{p}$.

MANUAL TÉCNICO DA VEGETAÇÃO BRASILEIRA. Rio de Janeiro: IBGE, 2012. 271p.

HONJI, R. M. et al. Biodiversidade e conservação da ictiofauna ameaçada de extinção da bacia do Rio Paraíba do Sul. Revista Biologia, [s.l], v. 17, p. 18-30, 2017.

IVANAUSKAS, N. M.; RODRIGUES, R. R.; NAVE, A. G. Aspectos ecológicos de um trecho de floresta de brejo em Itatinga, SP: florística, fitossociologia e seletividade de espécies. Brazilian Journal of Botany, São Paulo, v. 20, p. 139-153. 1997.

JOLY, C. A. Flooding tolerance in tropical trees. In: JACKSON, M. B.; DAVES, D. D.; LAMBERS, H. (eds.). Plant life under oxygen deprivation: ecology, physiology and biochemistry. SPB Academic Publishing, The Hague, Netherlands, p. 23-34.1991.

LEITE, E. C.; RODRIGUES, R. R. Fitossociologia e caracterização sucessional de um fragmento de Floresta Estacional no sudeste do Brasil. Revista Árvore, Viçosa, v. 32, p. 583-595. 2008.

Levantamento da Cobertura Vegetal Nativa do Bioma Mata Atlântica. In: BRASIL - Ministério do Meio Ambiente. Projeto de Conservação e Utilização Sustentável da Biodiversidade Brasileira - PROBIO: IESB, IGEO/UFRJ, UFF, Rio de Janeiro. 2014. Disponível em www.mma. gov.br/downloads. Acesso em 18 janeiro 2015.

LOBO, P. C.; JOLY, C. A. Aspectos ecofisiológicos da vegetação de mata ciliar do Sudeste do Brasil. In: RODRIGUES, R. R.; LEITÃO FILHO, H. F. (eds.). Matas ciliares: conservação e recuperação. Editora da Universidade de São Paulo, São Paulo, p. 143-157. 2000.

LOPES, S. F. et al. Canopy stratification in tropical seasonal forests: how the functional traits of community change among the layers. Bioscience Journal, Uberlândia, v. 30, p. 1551-1562. 2014.

LOPES, S. F. et al. An ecological comparison of floristic composition in seasonal semi deciduous forest in Southeast Brazil: implications for conservation. International Journal of Forestry Research, [s.l], v. 2012, p. 1-14. 2012.

MACIEL, A. B. C.; LIMA, Z. M. C.; DE MORAIS, A. C. S. Utilização da Trilha Ecológica como Instrumento de Educação Ambiental: Parque da Cidade Dom Nivaldo Monte, Natal/RN. Revista de Geociências do Nordeste, Caicó, v. 3, n. 2, p. 1-22, 2017. 
MAGURRAN, A. E. Ecological diversity and measurement. Princeton University Press, Princeton. 1988. 185p.

MARQUES, M. C. M.; SILVA, S. M.; SALINO, A. Florística e estrutura do componente arbustivoarbóreo de uma floresta higrófita da bacia do rio Jacaré-Pepira, SP, Brasil. Revista Brasileira de Botânica, São Paulo, v. 17, p. 495-506. 2003.

MARTINELLI, G.; MORAES, M. A. Livro vermelho da flora do Brasil. Jardim Botânico do Rio de Janeiro. 2013. Disponível em http://cncflora. jbrj. gov. br. Acesso em 28 jan. 2015.

MIRANDA, C. C.et al. Avaliação das preferências ecológicas de Clidemia urceolata em ecossistemas perturbados. Revista Árvore, Viçosa, v. 35, p. 1135-1144. 2011.

MAGALHÃES, J. E. P. O conflito socioambiental no horto florestal: um olhar pela educação ambiental crítica no programa elos de cidadania. Revista TransVersos, Rio de Janeiro, v. 7, n. 7, p. 100-133, 2016.

MUELLER-DOMBOIS, D.; ELLENBERG, H. Aims and methods of vegetation ecology. John Wiley \& Sons, New York. 1974. 547p.

MUSCARELLA, R.; FLEMING, T. H. The Role of Frugivorous Bats in Tropical Forest Succession. Biological Reviews, [s.1], v. 82, p. 573-590, 2007.

OKSANEN, J. et al. Vegan: Community Ecology Package. R package version 2.4-3.2017. Disponível em http://CRAN.R-project.org/package=vegan. Acesso em 5 maio 2017.

OLIVEIRA, J. A. Caracterização Física da Bacia do Ribeirão Cachimbal-Pinheiral, RJ e de suas principais paisagens degradadas. 1998. 142 f. Dissertação (Mestrado em Engenharia Florestal) Universidade Federal Rural do Rio de Janeiro, Seropédica. 1998.

RIO DE JANEIRO (Estado). Elaboração do Plano Estadual de Recursos Hídricos do Estado do Rio de Janeiro: R2F- Caracterização Ambiental. In: Fundação COPPETEC, INEA, SEA, Rio de Janeiro. 106p. 2014. Disponível em

http://www.inea.rj.gov.br/Portal/Agendas/GESTAODEAGUAS/InstrumentosdeGestodeRecHid/ PlanosdeBaciaHidrografica/index.htm\&lang=\#ad-image-0. Acesso em 28 junho 2015.

RIZZINI, C.T. Tratado de Fitogeografia do Brasil: aspectos ecológicos, sociológicos e florísticos. Âmbito Cultural Edições Ltda, Rio de Janeiro. 1997. 720p.

ROSA, S.F.; LONGHI, S.J.; LUDWIG, M. P. Aspectos florísticos e fitossociológicos da Reserva Capão de Tupanciretã, Tupanciretã, RS, Brasil. Ciência Florestal, Santa Maria, v. 18, p. 15-25. 2008.

ROTH, I. Stratification of a tropical forest as seen in dispersal types. Tasks for vegetation science, Dordrecht, Springer Netherlands, v. 17, p. 189. 1987.

ROZZA, A. F.; FARAH, F.T.; RODRIGUES, R. R. Ecological management of degraded forest fragments. In: RODRIGUES, R. R.; MARTINS, S. V. High Diversity Forest Restoration in Degraded Areas: Methods and Projects in Brazil. Nova Science Publishers, Nova York. 2006. 286p.

SILVA, A. C. et al. Florestas inundáveis: ecologia, florística e adaptações das espécies. Ed. UFLA, Lavras, 2012.167p.

SILVA, S. B. Levantamento florístico do componente arbustivo-arbóreo da vegetação ciliar de fragmento no rio parauapebas. Revista Agroecossistemas, [s.1], v. 9, n. 1, p. 99-115, 2017.

SZTUTMAN, M.; RODRIGUES, R. R. O mosaico vegetacional numa área de floresta contínua da planície litorânea, Parque Estadual da Campina do Encantado, Pariquera-Açu, SP. Revista Brasileira de Botânica, São Paulo, v. 25, p. 161-176.2002. 
TARGA, M. S.; ALMEIDA, A. A.; AlmEidA, J. C. R. Atores da restauração florestal do Vale do Paraíba. Repositório de Ciências Ambientais, [s.l], v. 1, n. 1, p. 1-5, 2017.

TCE-RJ. Tribunal de contas do Estado do Rio de Janeiro - Secretaria geral de planejamento. Estudo Sócio-Econômico de Pinheiral. Rio de Janeiro, 2004, 102p.

TEIXEIRA, A. P.; ASSIS, M. A. Caracterização florística e fitossociológica do componente arbustivo-arbóreo de uma floresta paludosa no Município de Rio Claro (SP), Brasil. Revista Brasileira de Botânica, São Paulo, v.28, n.3, p.467-476. 2005.

VAN DER PIJL, L. Principles of dispersal in higher plants. 3.ed. New York, Springer-Verlag, 1982.

VIDAL, M. M.; PIRES, M. M.; GUIMARAES, J. R. Large vertebrates as the missing components of seed-dispersal networks. Biological Conservation, [s.1], v. 163, p. 42-48. 2013.

YAMAMOTO, L.F.; KINOSHITA, L.S.; MARTINS, F. R. Síndromes de polinização e de dispersão em fragmentos da Floresta Estacional Semidecídua Montana, SP, Brasil. Acta Botanica Brasilica, Belo Horizonte, v. 21, p. 553-573. 2007. 